\title{
RKI-1447, a Rho-kinase inhibitor causes ocular hypotension, actin stress fiber disruption and increased phagocytosis
}

Yalong Dang, MD, Ph.D. ${ }^{1}$, Chao Wang, MB ${ }^{1,2}$, Priyal Shah, BSc ${ }^{1,3}$, Susannah Waxman, BA ${ }^{1}$, Ralitsa T. Loewen, $\mathrm{MD}^{1}$, and Nils A. Loewen, MD, Ph.D. ${ }^{* 1}$

1. Department of Ophthalmology, University of Pittsburgh School of Medicine, Pittsburgh, Pennsylvania, United States of America

2. Department of Ophthalmology, Xiangya Hospital, Central South University, Changsha, Hunan, People's Republic of China

3. Institute of Ophthalmology and Visual Science, Rutgers New Jersey Medical School, Newark, New Jersey, United States of America.

\section{* Corresponding author}

Nils A. Loewen, MD, Ph.D.

203 Lothrop St, Suite 819, Pittsburgh, PA 15213

Email: loewen.nils@gmail.com, Phone (fax): 412-605-1541 


\begin{abstract}
Purpose: This study investigated the hypotensive effect of RKI-1447, a Rho kinase inhibitor, in a porcine ex vivo pigmentary glaucoma model.

Methods: Twenty-eight porcine anterior chambers were perfused with medium supplemented with $1.67 \times 10^{7}$ pigment particles/ml for 48 hours before treatment with RKI-1447 $(n=16)$ or vehicle control $(n=12)$. Intraocular pressure (IOP) was recorded and outflow facility was calculated. Primary trabecular meshwork cells were exposed to RKI-1447 or vehicle control; effects on the cytoskeleton, motility, and phagocytosis were evaluated.

Result: Compared to baseline, the perfusion of pigment caused a significant increase in IOP in the RKI-1447 group ( $P=0.003$ ) at 48 hours. Subsequent treatment with RKI-1447 significantly reduced IOP from $20.14+/-2.59$ $\mathrm{mmHg}$ to $13.38+/-0.91 \mathrm{mmHg}(P=0.02)$. Pigment perfusion reduced the outflow facility from $0.27+/-0.03$ at baseline to $0.18+/-0.02$ at 48 hours $(P<0.001)$. This was partially reversed with RKI-1447. RKI-1447 caused no apparent histological changes in the micro- or macroscopic TM appearance. RKI-1447-treated primary TM cells showed significant disruption of the actin cytoskeleton both in the presence and absence of pigment $(P<0.001)$ but no effect on TM migration was observed. Pigment-treated TM cells exhibited a reduction in TM phagocytosis, which RKI-1447 reversed.
\end{abstract}

Conclusion: RKI-1447 significantly reduces IOP by disrupting TM stress fibers and increasing TM phagocytosis. These features may make it useful for the treatment of secondary glaucomas with an increased phagocytic load.

Keywords: Pigmentary glaucoma, ROCK kinase inhibitor, RKI-1447, Trabecular meshwork, Phagocytosis, Cytoskeleton. 


\section{Introduction}

Ocular hypertension frequently observed in glaucoma patients exhibits a complex pathomechanism that remains incompletely understood [1]. Intraocular pressure (IOP) remains the single most important modifiable variable in glaucoma treatment [2]. Current topical medications for glaucoma typically increase the uveoscleral outflow of aqueous humor (prostaglandin analogs) or reduce aqueous humor production (betaadrenergic blockers, alpha-adrenergic agonists, carbonic anhydrase inhibitors). Until recently, miotics were the only class that increases the conventional outflow as the primary mechanism but were hampered by significant side effects that include ciliary spasm and dim vision caused by miosis [2]. Newly introduced nitric oxide (NO) donors increase the facility by relaxing the TM [3] and the distal outflow tract [4, 5]. Rho-kinase inhibitors also relax the TM cytoskeleton [6, 7]. In the present study, we examine the hypotensive effect of RKI-1447, which was originally introduced as an anti-tumor agent in breast cancer treatment [8].

Rho-associated protein kinase (ROCK) is part of the serine-threonine kinases that act on the cytoskeleton to regulate the movement and shape of cells. It plays a central role in biological functions including stress-fiber formation and contraction, cell adhesion, migration and invasion, transformation, phagocytosis and apoptosis, cytokinesis and mitosis as well as differentiation $[9,10]$. Previous studies found that ROCK is expressed in the TM and upregulated in the optic nerve head of glaucomatous eyes [11, 12]. Honjo and colleagues found the ROCK inhibitor Y-27632 increased outflow facility in a dose-dependent manner in rabbit eyes [13]. The investigators suggested that the hypotensive effect may result from a disruption of actin bundles and reduction in focal adhesion of TM cells [13]. Similarly, recent studies found that ROCK inhibitors disrupt tight junctions thereby increasing the permeability of Schlemm's canal endothelium $[14,15]$ in addition to decreasing the episcleral venous pressure [16].

Ripasudil (K-115) was the first approved ROCK inhibitor for glaucoma [6, 7]. Two randomized trials involving 413 Japanese primary open-angle glaucoma patients found that Ripasudil achieved an average of 0.4-1.6 $\mathrm{mmHg}$ larger IOP reduction than the administration of timolol or latanoprost alone [17]. Phase II/III trials that aim to investigate the IOP-lowering effect of four different ROCK inhibitors (AMA0076 [18], AR13324 [19], K-115 [7] and PG-324 [20]) are currently in process. Several trials that employed functionally and structurally similar compounds were discontinued due to the low therapeutic efficiency and high incidence of adverse events [21].

First characterized by Patel et al. in 2012 [8], RKI-1447 inhibits the type I kinase by binding to the ATP binding site through interaction with the Asp-Phe-Gly motif and hinge region (Figure 1) [8, 22]. In vitro, it selectively inhibits ROCK1/2-mediated actin stress fiber formation at a low IC-50 (14.5 nM and 6.2 nM for ROCK1 and ROCK2, respectively [23]) following lysophosphatidic acid stimulation. Unlike other ROCK inhibitors, RKI-1447 does not exhibit cross-reactivity with AKT, MEK, PKA, and PKG signaling, even at a high concentration of up to $10 \mu \mathrm{M}[8,23]$, reducing the risk of side effects. Recently, our group developed an ex vivo pigmentary glaucoma model that exhibited IOP elevation, typical TM stress fiber formation, and reduction in TM phagocytosis [24, 25]. In this paper, we examine the ocular hypotensive effect of RKI-1447 in an ex vivo anterior chamber perfusion model and investigate its possible mechanisms. 


\section{Materials and Methods}

\section{Pig eye perfusion, pigmentary glaucoma model, and RKI-1447 intervention}

Because eyes were obtained from an abattoir, no Institutional Animal Care and Use Committee protocol was required. "Porcine anterior segment perfusion culture is a well-established homolog to human and primate anterior segment culture, with porcine outflow tracts being similar in anatomy and more readily attainable within short time-periods after death $[26,27] . "$ The setup of the porcine eye perfusion system followed our previous protocols [24, 28-30]. Briefly, 40 freshly enucleated pig eyes were obtained from a local slaughterhouse (Thoma Meat Market, Saxonburg, PA) and processed within two hours of sacrifice. After the removal of extraocular tissues, the eyes were disinfected in a $5 \%$ povidone-iodine ophthalmic solution (betadine 5\%; Alcon, Fort Worth, TX, USA) for two minutes, washed three times with PBS, and hemisected. Anterior chambers were then mounted on the perfusion dishes and subsequently underwent continuous perfusion with 1\% FBS supplemented with DMEM and antibiotics at a constant rate of $3 \mu \mathrm{l}$ per min.

Once baseline IOPs were obtained, 28 of the 40 eyes were perfused with medium supplemented with $1.67 \times 10^{7}$ pigment particles $/ \mathrm{ml}$ for 48 hours before being subjected to the RKI-1447 ( $n=16$ ) or the vehicle control $(n=12)$. For the RKI-1447 group, RKI-1447 was diluted into the pigment medium to a final concentration of $1 \mu \mathrm{M}$. For the vehicle control, eyes were continuously perfused with the same pigment medium. A total of 12 of the 40 samples without pigment perfusion and RKI-1447 served as the normal control. IOPs were measured using a pressure transducer at two-minute intervals.

\section{Production of pigment dispersion particles}

Pigment dispersion particles were produced as previously described with a freeze-thaw protocol [31, 32]. In brief, ten porcine irises were sealed in a 50- $\mathrm{ml}$ tube, frozen at a temperature of $-80^{\circ}$ for 2 hours, then thawed at room temperature. After two cycles of freeze-thaw, $15 \mathrm{ml}$ of phosphate-buffered saline (PBS) was added to the tube, and the mixture was pipetted up and down 100 times with a $3 \mathrm{ml}$ Pasteur pipette before straining through a 70- $\mu \mathrm{m}$ cell strainer (cat\#431751, Corning Incorporated, Durham, NC, USA). The suspension was centrifuged at $3000 \mathrm{rpm}$ for $15 \mathrm{~min}$. The supernatant was discarded, and the pigment pellet was resuspended in PBS and centrifuged again at the same settings. Finally, pigment particles were resuspended in $4 \mathrm{ml}$ of PBS as the stock solution and titered using a hemocytometer (\#1490, Hausser Scientific, Horsham, PA) at 1000 -fold dilution as per the method previously described [32].

\section{Histology}

At the conclusion of the experiments, the anterior segments were dismounted, washed with PBS, fixed with 4\% PFA for 24 hours and embedded in paraffin. Samples were sectioned at 6-micron thickness, followed by staining with hematoxylin and eosin (H\&E).

\section{Primary TM culture}

Primary porcine TM cells were isolated and characterized as described before [32]. A porcine anterior segment was dissected by removing the sclera and cornea and creating TM strips of $1 \mathrm{~mm}$. Three pieces were cultured in a T75 flask with TM culture media containing 5\% FBS supplemented with Opti MEM (31985-070, Gibco, Life Technologies, Grand Island, NY) and antibiotics (15240062, Thermo Fisher Scientific, Waltham, MA). The TM cells had migrated onto the bottom of the flask by day four and formed clones. Cells were passaged at $80 \%$ confluence and replated at a 1:3 ratio. We characterized these cells by immunostaining with a combination of TM specific antibodies (alpha smooth muscle actin [alpha-SMA] antibody, matrix gla protein 
[MGP] antibody, and aquaporin 1 [AQP1]), an in vitro phagocytic assay and myocilin induction by glucocorticosteroids [32]. To avoid transformation, differentiation, and senescence, only cells with up to four passages were used.

\section{Immunostaining}

For further characterization, we plated the TM cells onto $10 \times 10 \mathrm{~mm}$ glass slides and fixed them with $4 \%$ PFA at $80 \%$ confluence. This was followed by washing with PBS three times and incubation with the primary antibody overnight at $4^{\circ} \mathrm{C}$. Primary antibodies were goat polyclonal anti-MGP antibodies (1:100 dilution, sc32820, Santa Cruz, Dallas, Texas), rabbit polyclonal anti alpha-SMA (1:100, ab5694, Abcam, Cambridge, MA) and rabbit polyclonal anti-AQP1 antibodies (1:100, Sc-20810, Santa Cruz, Dallas, Texas). After three rinses in PBS, a mixture of secondary antibodies (donkey-anti-goat Alexa Fluor ${ }^{\circledR} 647$ [1: 200, ab150131, Abcam, Cambridge, MA] and goat anti-rabbit Alexa Fluor ${ }^{\circledR} 488$ [1:1000, A27034, Thermo Scientific, Waltham, MA]) was incubated with the cells for 45 minutes at room temperature. Cell nuclei were counterstained with DAPI (1: 1000, D1306, Thermo Fisher Scientific, Waltham, MA) for 15 minutes. Images were taken using an upright laser scanning confocal microscope at 400x magnification (BX61, Olympus, Tokyo, Japan).

To quantify the stress fiber formation, cells were fixed with 4\% PFA for 24 hours before being stained with Alexa Fluor ${ }^{\circledR} 488$ Phalloidin for 30 minutes (1:20, A12379, Thermo Scientific, Waltham, MA). Cell nuclei were labeled with 4',6-diamidino-2-phenylindole (DAPI). Ten randomized visual fields were chosen to quantify the percentages of cells with stress fibers for each group.

\section{TM cell motility assay}

Evaluation of in vitro TM cell motility utilized a scratch assay with a minor modification[33]. In brief, $2 \times 10^{5}$ primary TM cells were seeded into each well of a six-well plate and cultured in an incubated chamber equipped with a digital camera and image acquisition software. After reaching $80 \%$ confluence cells were treated with pigment particles at a concentration of $1.67 \times 10^{7}$ particles $/ \mathrm{ml}$ for 24 hours. A $10 \mu$ l pipette tip (info) was used to create a cell-free scratch that had a consistent width. RKI-1447 (final concentration: $1 \mu \mathrm{M}$ ) or vehicle was applied. Pictures from ten positions of each well were acquired at 40-fold magnification in twohour intervals for 20 hours. The numbers of TM cells which moved into the cell-free scratch were counted.

\section{TM cell phagocytosis assay}

Phagocytic activity of the primary TM cells was measured by flow cytometry using a fluorescent microsphere ingestion test [29]. $2 \times 10^{5}$ primary TM cells were seeded into each well of a six-well plate and exposed to $1.67 \times 10^{7}$ pigment particles $/ \mathrm{ml}$ or vehicle treatment for seven days. Half of the cells were then treated with $1 \mu \mathrm{M}$ RKI-1447 for 24 hours while the rest underwent sham treatment. To quantify the phagocytic activity, cells were incubated with $5 \times 10^{8} 0.5$-micron fluorescent microspheres per milliliter at $37^{\circ} \mathrm{C}$ for 1 hour. After washing thoroughly with PBS, cells were dissociated with trypsin and resuspended in $200 \mu \mathrm{IBS}$. The percentage of cells which had ingested microspheres was determined by flow cytometry.

\section{Statistics}

Quantitative data were presented as the mean +/- standard error and processed by PASW 18.0 (SPSS Inc., Chicago, IL, USA). A paired t-test was used to compare IOP changes from baseline. Other quantitative data were compared by one-way ANOVA. A $p \leq 0.05$ was considered statistically significant. 


\section{Results}

\section{IOP reduction and increase of outflow facility}

Baseline IOPs among these three groups were comparable $(10.32+/-0.82 \mathrm{mmHg}$ in the pigment group versus $12.21+/-1.06 \mathrm{mmHg}$ in the RKI-1447 group and $10.21+/-1.15 \mathrm{mmHg}$ in the control group, $\mathrm{P}=0.291$ ). Compared to each baseline, the intracameral perfusion of pigment particles resulted in a significant IOP elevation in the pigment group $(\mathrm{P}=0.038)$ and the RKI-1447 group $(\mathrm{P}=0.003)$ at 48 hours, while IOPs in the control group remained unchanged $(P=0.108)$. RKI-1447 significantly reduced IOP from $20.14+/-2.59 \mathrm{mmHg}$ to $13.38+/-0.91 \mathrm{mmHg}$ at 60 hours and remained at this low level throughout the study. In contrast, IOP in the pigment group remained high in comparison to its baseline (all $P<0.05$ ). At all time points after RKI-1447, IOP in the RKI-1447 group exhibited no statistical difference compared to the control (all P>0.05); however, both were significantly lower than those in the pigment group (all $\mathrm{P}<0.05$ ) (Figure $2 \mathrm{~A}$ ).

Because the episcleral venous pressure is close to zero in the perfusion system, outflow facility is inversely correlated with IOP. In the current study, baseline outflow facility exhibited no difference across the three groups $(P=0.316)$. Pigment perfusion caused a significant reduction in outflow facility in the RKI-1447 group, from $0.27+/-0.03$ at the baseline to $0.18+/-0.02$ at 48 hours $(P<0.001)$, while RKI- 1447 partially reversed this effect by $14.57 \%$ to $48.35 \%$ at the follow-up time points. In contrast, outflow facility in the pigment group continuously decreased from a baseline of $0.31+/-0.03$ to $0.15+/-0.01$ at the end of the study (all $P<0.05$, compared to its baseline). In the control group, outflow facility exhibited a slight decrease as time elapsed (Fig. 2 B).

Normal TM exhibits a light-pigmented, multilayer, strainer-like structure connected with the aqueous plexus (Figure 3a). Consistent with our previous findings [24, 29], pigment perfusion resulted in an increased pigmentation in the TM, while most of the pigment particles seemed to be phagocytosed by TM cells without physically obstructing the TM (Figure 3b). RKI-1447 treatment exhibited no apparent changes in TM histology (Figure 3c).

\section{Disruption of TM cell actin stress fibers}

The primary TM cells had an elongated morphology (Figure 4a), active phagocytosis (Figure 4b), and were positive for TM markers alpha-SMA, AQP1, and MGP (Figures $\mathbf{4 c}$ and $4 \mathbf{4 d}$ ). Pigment dispersion did not cause an apparent morphological change in TM cells [29] (Figures 4e and 4g), while RKI-1447 stimulated a cell body contraction within minutes, in the presence or absence of pigment exposure (Figures $4 \mathrm{f}$ and $\mathbf{4 h}$ ).

F-actin cytoskeleton labeling with Alexa Fluor ${ }^{\circledast} 488$ Phalloidin, showed a baseline of $32.57 \pm 2.66 \%$ actin stress fibers in normal TM cells, compared to $67.13 \pm 4.00 \%$ in the pigment-exposed TM cells $(P<0.001)$ (Figures $4 \mathbf{i}$ and $4 \mathrm{k})$. RKI-1447 abolished these stress fibers, both in normal TM cells $(2.57 \pm 0.86 \%$ versus $32.57 \pm 2.66 \%, P<0.001)$ and pigment-exposed TM cells $(2.51+/-0.71 \%$ versus $67.13 \pm 4.00 \%, P<0.001)$ (Figures $4 \mathrm{j}$, 4i, and $4 \mathrm{~m}$ ). 


\section{TM cell motility}

Because TM cells of glaucomatous eyes [29, 34] or TM cells exposed to pigment dispersion [32], have altered motility, we tested the effect of RKI-1447 on motility using a modified scratch assay. As shown in Figure 5 , the pigment exposure statistically decreased the numbers of migrating TM cells $(60.83 \pm 3.46$ in the normal control versus $31.75 \pm 3.36$ cells in the pigment treated group, $\mathrm{P}<0.001$ ) while RKI-1447 seemed to have no effect on the TM migration, either for the normal TM cells or the pigment-treated TM cells ( $P=0.091$ and $\mathrm{P}=0.398$, compared to each baseline).

As we have seen before [24, 32], pigment exposure caused a reduction in TM phagocytosis by $10.31 \%$ while RKI-1447 reversed this effect and increased it to $111.52 \%$ in comparison to the normal baseline.

\section{Discussion}

Pigmentary glaucoma (PG) is a common type of secondary open-angle glaucoma with an incidence of 1.4 per $100,000[35,36]$. A readily recognizable feature of this disease is the dispersion of pigment onto intraocular structures, especially on the corneal endothelium and the trabecular meshwork [37]. Our previous study in a porcine eye perfusion model suggested that iris pigment particles induced TM stress fibers, reduced TM phagocytosis, adhesion, and motility, and caused an IOP elevation [29]. In the current study, we found that RKI-1447, a ROCK1/ROCK2 inhibitor, efficiently reduced the IOP by $33.58 \%$ and increased the outflow facility by $38.25 \%$ in the same pigmentary glaucoma model. The in vitro results suggest these changes result from an extensive disruption of TM actin stress fibers. In the current study, we utilized a porcine anterior segment perfusion model that mimics the TM changes and IOP phenotype of human PG to examine the hypotensive effect of RKI-1447, a drug previously used in breast cancer treatment [8]. Pigment dispersion caused a significant IOP elevation that plateaued at $48 \mathrm{hrs}$, and this was partially reversed by RKI-1447. Cytoskeletal changes of TM cells, their stiffness, adhesion, migration, contraction, and phagocytosis can all impact the outflow facility [38, 39], and ROCK signaling plays a central role in all these [11, 40].

The impact of the actin cytoskeleton and cell or extracellular matrix stiffness on aqueous outflow facility has been well established [41, 42]. Consistent with our previous work [29], only a small portion of stress fibers formed in normal control TM while pigment exposure caused a 2.06-fold increase. Actin stress fibers contribute to the stiffness of both the TM cells and the extracellular matrix that surrounds them $[41,43,44]$ which directly impact aqueous outflow facility [42]. Gavara et al. also found that the presence of aligned and thick stress fibers in the cell periphery gave rise to reinforced cell stiffness [45]. In general, factors that induce stress fiber formation increase TM stiffness and decrease outflow facility as is the case with senescence and aging, risk factors for primary open-angle glaucoma [46]. In a different study, dexamethasone, an inducer of cross-linked actin networks $[47,48]$, was shown to increase in three days the stiffness of primary TM cells twofold and that of extracellular fourfold [44]. Clark et al. observed an IOP elevation in ex vivo perfused human anterior segments [48]. In contrast, several reagents that disrupt actin stress fibers exhibit an ocular hypotensive effect. For instance, $\mathrm{Y} 27632$ has been found to abolish cyclic stress-induced stress fiber and increase the facility by $40 \%-80 \%$ in pig eyes [49]. Other molecules that target the myosin light chain and its kinase exhibited a similar effect [50-52]. In the current study, RKI-1447 significantly disrupted stress fibers in pigment-treated TM cells, and the IOP-reducing effect could be at least partially attributed to this. 
As is the case with other ROCK inhibitors [13, 52, 53], we found RKI-1447 caused TM cell contraction, rounding and decreased adhesion. Live cell imaging indicated that pigment exposure reduces TM cell migration, but this was unaffected by RKI-1447, suggesting that the IOP-lowering effect might not be directly related to TM motility.

Phagocytosis requires a well-coordinated rearrangement of the actin cytoskeleton [54, 55]. TM cells can phagocytose debris to prevent it from entering the outflow tract $[38,56]$. A reduction in TM phagocytosis has been observed in different types of glaucoma and glaucoma models. For instance, perfusion of human anterior segments with dexamethasone for 21 days resulted in a 57\% decrease of TM phagocytosis without TM cell loss [57]. Switching B-Cell Complex 70 kDa Subunit (SWAP70), vasodilator-stimulated phosphoprotein (VASP), and Wiskott-Aldrich Syndrome protein (WASp), proteins involved in actin polymerization and phagocytosis, were believed to play an essential role in this process [32, 58]. Our observation here of a $10.3 \%$ reduction in TM phagocytosis following pigment exposure and increase an 11.5\% increase by RKI-1447 matches this.

Our study had several limitations. The anatomical difference between the human and the porcine outflow tract and their angular aqueous plexus is significant [59]. Domestic pigs, even breeders with a longer lifespan, do not typically develop glaucoma. A study by Camras et al. suggested that TM stiffness was correlated with outflow facility in human eye perfusion but not in the pig specimens [60]. This ex vivo study does not account for critical factors that might only be detectable in vivo. It is possible that RKI-1447 does not only have an impact on the TM but also causes a reduction of post-trabecular outflow resistance $[4,5]$.

In conclusion, the ROCK inhibitor RKI-1447 disrupted TM stress fibers, increased phagocytosis and lowered IOP in an ex vivo model of pigmentary glaucoma.

\section{Acknowledgments}

We acknowledge support from the National Eye Institute K08EY022737 (NL), P30 EY08098 to the Department of Ophthalmology, from the Wiegand Fellowship of the Eye and Ear Foundation (YD), from the Initiative to Cure Glaucoma of the Eye and Ear Foundation of Pittsburgh (NAL), from an unrestricted grant from Research to Prevent Blindness, New York, NY and an unrestricted grant from the Third Xiangya Hospital of Central South University for studying at the University of Pittsburgh (CW). We thank Vappingo (Moo Media Limited, UK) for editing the English text of this manuscript.

\section{Conflict of interest}

The authors declare that they have no conflict of interest. 


\section{References}

1. Janssen SF, Gorgels TGMF, Ramdas WD, et al (2013) The vast complexity of primary open angle glaucoma: disease genes, risks, molecular mechanisms and pathobiology. Prog Retin Eye Res 37:31-67

2. Weinreb RN, Aung T, Medeiros FA (2014) The pathophysiology and treatment of glaucoma: a review. JAMA 311:1901-1911

3. Weinreb RN, Ong T, Scassellati Sforzolini B, et al (2014) A randomised, controlled comparison of latanoprostene bunod and latanoprost $0.005 \%$ in the treatment of ocular hypertension and open angle glaucoma: the VOYAGER study. Br J Ophthalmol bjophthalmol-2014

4. Waxman S, Wang C, Dang Y, et al (2018) Structure-Function Changes of the Distal Outflow Tract in Response to Nitric Oxide. Preprint

5. McDonnell F, Dismuke WM, Overby DR, Stamer WD (2018) PHARMACOLOGICAL REGULATION OF OUTFLOW RESISTANCE DISTAL TO SCHLEMM'S CANAL. Am J Physiol Cell Physiol. doi: 10.1152/ajpcell.00024.2018

6. Garnock-Jones KP (2014) Ripasudil: first global approval. Drugs 74:2211-2215

7. Tanihara $\mathrm{H}$, Inoue $\mathrm{T}$, Yamamoto $\mathrm{T}$, et al (2016) One-year clinical evaluation of $0.4 \%$ ripasudil (K-115) in patients with open-angle glaucoma and ocular hypertension. Acta Ophthalmol 94:e26-34

8. Patel RA, Forinash KD, Pireddu R, et al (2012) RKI-1447 is a potent inhibitor of the Rho-associated ROCK kinases with anti-invasive and antitumor activities in breast cancer. Cancer Res 72:5025-5034

9. Riento K, Ridley AJ (2003) Rocks: multifunctional kinases in cell behaviour. Nat Rev Mol Cell Biol 4:446456

10. Katoh K, Kano Y, Ookawara S (2007) Rho-kinase dependent organization of stress fibers and focal adhesions in cultured fibroblasts. Genes Cells 12:623-638

11. Wang J, Liu X, Zhong Y (2013) Rho/Rho-associated kinase pathway in glaucoma (Review). Int J Oncol 43:1357-1367

12. Goldhagen B, Proia AD, Epstein DL, Rao PV (2012) Elevated levels of RhoA in the optic nerve head of human eyes with glaucoma. J Glaucoma 21:530-538

13. Honjo $\mathrm{M}$, Tanihara $\mathrm{H}$, Inatani $\mathrm{M}$, et al (2001) Effects of rho-associated protein kinase inhibitor $\mathrm{Y}-27632$ on intraocular pressure and outflow facility. Invest Ophthalmol Vis Sci 42:137-144

14. Kameda $\mathrm{T}$, Inoue $\mathrm{T}$, Inatani $\mathrm{M}$, et al (2012) The Effect of Rho-Associated Protein Kinase Inhibitor on Monkey Schlemm's Canal Endothelial CellsEffects of ROCK Inhibitor on Schlemm's Canal Cells. Invest Ophthalmol Vis Sci 53:3092-3103

15. Kaneko Y, Ohta M, Inoue T, et al (2016) Effects of K-115 (Ripasudil), a novel ROCK inhibitor, on trabecular meshwork and Schlemm's canal endothelial cells. Sci Rep 6:19640

16. Kiel JW, Kopczynski CC (2015) Effect of AR-13324 on episcleral venous pressure in Dutch belted rabbits. J Ocul Pharmacol Ther 31:146-151 
17. Tanihara H, Inoue T, Yamamoto T, et al (2015) Additive Intraocular Pressure-Lowering Effects of the Rho Kinase Inhibitor Ripasudil (K-115) Combined With Timolol or Latanoprost: A Report of 2 Randomized Clinical Trials. JAMA Ophthalmol 133:755-761

18. Multiple Dose-parallel-group Study of AMA0076 in Patients With Primary Open-Angle Glaucoma or Ocular Hypertension - Full Text View - ClinicalTrials.gov. https://clinicaltrials.gov/ct2/show/NCT02136940. Accessed 7 Sep 2017

19. Bacharach J, Dubiner HB, Levy B, et al (2015) Double-masked, randomized, dose-response study of AR13324 versus latanoprost in patients with elevated intraocular pressure. Ophthalmology 122:302-307

20. Double-masked Study of PG324 Ophthalmic Solution in Patients With Open-angle Glaucoma or Ocular Hypertension - Full Text View - ClinicalTrials.gov. https://clinicaltrials.gov/ct2/show/NCT02674854. Accessed 7 Sep 2017

21. Wang SK, Chang RT (2014) An emerging treatment option for glaucoma: Rho kinase inhibitors. Clin Ophthalmol 8:883-890

22. Martin MP, Zhu J-Y, Schonbrunn E (2012) Rho-associated protein kinase 1 (ROCK 1) IN COMPLEX WITH RKI1447

23. RKI-1447 | ROCK inhibitor | Read Reviews \& Product Use Citations. http://www.selleckchem.com/products/rki1447.html?gclid=CjOKEQjw3MPNBRDjOYztg4LGvOcBEiQA5hTrHORIVz8jg86KuAdDjJRNteNbuGISVI77ZSILD 2KVnPkaArcO8P8HAQ. Accessed 7 Sep 2017

24. Dang Y, Waxman S, Wang C, et al (2017) Intraocular pressure elevation precedes a phagocytosis decline in a model of pigmentary glaucoma. bioRxiv 175695

25. Dang Y, Waxman S, Wang C, et al (2017) Trabecular Meshwork Failure In A Model Of Pigmentary Glaucoma. bioRxiv 118448

26. McMenamin PG, Steptoe RJ (1991) Normal anatomy of the aqueous humour outflow system in the domestic pig eye. J Anat 178:65-77

27. Ruiz-Ederra J, García M, Hernández M, et al (2005) The pig eye as a novel model of glaucoma. Exp Eye Res 81:561-569

28. Dang Y, Waxman S, Wang C, et al (2017) Freeze-thaw decellularization of the trabecular meshwork in an ex vivo eye perfusion model. PeerJ Preprints

29. Dang $Y$, Waxman S, Wang C, et al (2017) A porcine ex vivo model of pigmentary glaucoma. bioRxiv 118448

30. Loewen RT, Roy P, Park DB, et al (2016) A Porcine Anterior Segment Perfusion and Transduction Model With Direct Visualization of the Trabecular Meshwork. Invest Ophthalmol Vis Sci 57:1338-1344

31. Dang $\mathrm{Y}$, Waxman S, Wang C, et al (2017) Freeze-thaw decellularization of the trabecular meshwork in anex vivoeye perfusion model. PeerJ 5:e3629

32. Dang Y, Waxman S, Wang C, et al (2018) A porcine ex vivo model of pigmentary glaucoma. Sci Rep 8:5468 
33. Wang G-Q, Dang Y-L, Huang Q, et al (2017) In Vitro Evaluation of the Effects of Intraocular Lens Material on Lens Epithelial Cell Proliferation, Migration, and Transformation. Curr Eye Res 42:72-78

34. Hogg P, Calthorpe M, Batterbury M, Grierson I (2000) Aqueous humor stimulates the migration of human trabecular meshwork cells in vitro. Invest Ophthalmol Vis Sci 41:1091-1098

35. Siddiqui Y, Ten Hulzen RD, Cameron JD, et al (2003) What is the risk of developing pigmentary glaucoma from pigment dispersion syndrome? Am J Ophthalmol 135:794-799

36. Ritch R, Steinberger D, Liebmann JM (1993) Prevalence of pigment dispersion syndrome in a population undergoing glaucoma screening. Am J Ophthalmol 115:707-710

37. Qing G, Wang N, Tang X, et al (2009) Clinical characteristics of pigment dispersion syndrome in Chinese patients. Eye 23:1641-1646

38. Llobet A, Gasull X, Gual A (2003) Understanding trabecular meshwork physiology: a key to the control of intraocular pressure? News Physiol Sci 18:205-209

39. Abu-Hassan DW, Acott TS, Kelley MJ (2014) The Trabecular Meshwork: A Basic Review of Form and Function. J Ocul Biol Dis Infor 2:

40. Nakajima E, Nakajima T, Minagawa Y, et al (2005) Contribution of ROCK in contraction of trabecular meshwork: proposed mechanism for regulating aqueous outflow in monkey and human eyes. J Pharm Sci 94:701-708

41. Doornaert B, Leblond V, Planus E, et al (2003) Time course of actin cytoskeleton stiffness and matrix adhesion molecules in human bronchial epithelial cell cultures. Exp Cell Res 287:199-208

42. Wang K, Read AT, Sulchek T, Ethier CR (2017) Trabecular meshwork stiffness in glaucoma. Exp Eye Res 158:3-12

43. Calzado-Martín A, Encinar M, Tamayo J, et al (2016) Effect of Actin Organization on the Stiffness of Living Breast Cancer Cells Revealed by Peak-Force Modulation Atomic Force Microscopy. ACS Nano 10:33653374

44. Raghunathan VK, Morgan JT, Park SA, et al (2015) Dexamethasone Stiffens Trabecular Meshwork, Trabecular Meshwork Cells, and Matrix. Invest Ophthalmol Vis Sci 56:4447-4459

45. Gavara N, Chadwick RS (2016) Relationship between cell stiffness and stress fiber amount, assessed by simultaneous atomic force microscopy and live-cell fluorescence imaging. Biomech Model Mechanobiol 15:511-523

46. Morgan JT, Raghunathan VK, Chang Y-R, et al (2015) The intrinsic stiffness of human trabecular meshwork cells increases with senescence. Oncotarget 6:15362-15374

47. Peng J, Feng $X-Y$, Ye Z-M, et al (2016) Effects of dexamethasone and HA1077 on actin cytoskeleton and $\beta$ catenin in cultured human trabecular meshwork cells. Int J Ophthalmol 9:1376-1380

48. Clark AF, Brotchie D, Read AT, et al (2005) Dexamethasone alters F-actin architecture and promotes cross-linked actin network formation in human trabecular meshwork tissue. Cell Motil Cytoskeleton 60:83-95 
49. Rao PV, Deng PF, Kumar J, Epstein DL (2001) Modulation of aqueous humor outflow facility by the Rho kinase-specific inhibitor Y-27632. Invest Ophthalmol Vis Sci 42:1029-1037

50. Rao PV, Deng P, Sasaki Y, Epstein DL (2005) Regulation of myosin light chain phosphorylation in the trabecular meshwork: role in aqueous humour outflow facility. Exp Eye Res 80:197-206

51. Zhang M, Rao PV (2005) Blebbistatin, a novel inhibitor of myosin II ATPase activity, increases aqueous humor outflow facility in perfused enucleated porcine eyes. Invest Ophthalmol Vis Sci 46:4130-4138

52. Honjo $\mathrm{M}$, Inatani $\mathrm{M}$, Kido $\mathrm{N}$, et al (2002) A myosin light chain kinase inhibitor, ML-9, lowers the intraocular pressure in rabbit eyes. Exp Eye Res 75:135-142

53. Rao PV, Deng P, Maddala R, et al (2005) Expression of dominant negative Rho-binding domain of Rhokinase in organ cultured human eye anterior segments increases aqueous humor outflow. Mol Vis 11:288-297

54. May RC, Machesky LM (2001) Phagocytosis and the actin cytoskeleton. J Cell Sci 114:1061-1077

55. Mannherz HG (2017) The Actin Cytoskeleton and Bacterial Infection. Springer

56. Rohen JW, van der Zypen E (1968) The phagocytic activity of the trabecular meshwork endothelium. Albrecht von Graefes Arch Klin Ophthalmol 175:143-160

57. Matsumoto Y, Johnson DH (1997) Dexamethasone decreases phagocytosis by human trabecular meshwork cells in situ. Invest Ophthalmol Vis Sci 38:1902-1907

58. Castellano F, Le Clainche C, Patin D, et al (2001) A WASp-VASP complex regulates actin polymerization at the plasma membrane. EMBO J 20:5603-5614

59. McMenamin PG, Steptoe RJ (1991) Normal anatomy of the aqueous humour outflow system in the domestic pig eye. J Anat 178:65-77

60. Camras LJ, Stamer WD, Epstein D, et al (2012) Differential effects of trabecular meshwork stiffness on outflow facility in normal human and porcine eyes. Invest Ophthalmol Vis Sci 53:5242-5250 


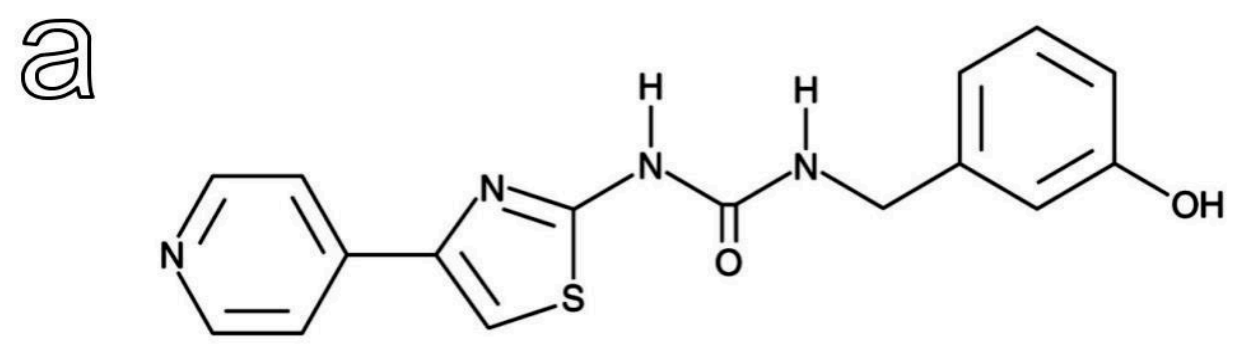

ڤ)

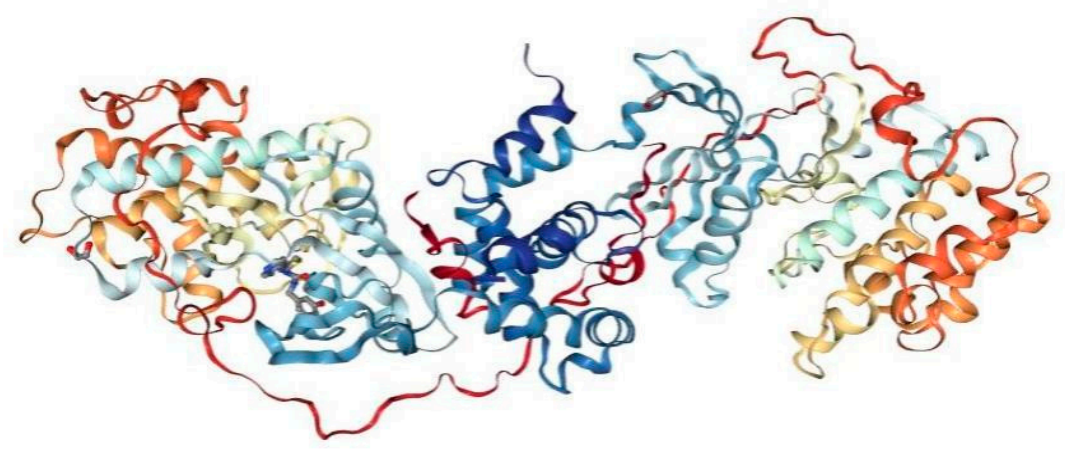

Figure 1. Molecular structure of RKI-1447. (a) RKI-1447 is a cell-permeable pyridyl thiazolyl-urea that acts as an ATP site-targeting Rho kinase inhibitor with an IC50 of $14.5 \mathrm{nM}$ and $6.2 \mathrm{nM}$ for ROCK1 and ROCK2, respectively. (b) RKI-1447 interacts with the Asp-Phe-Gly motif and hinge region of ROCKs (blue). 


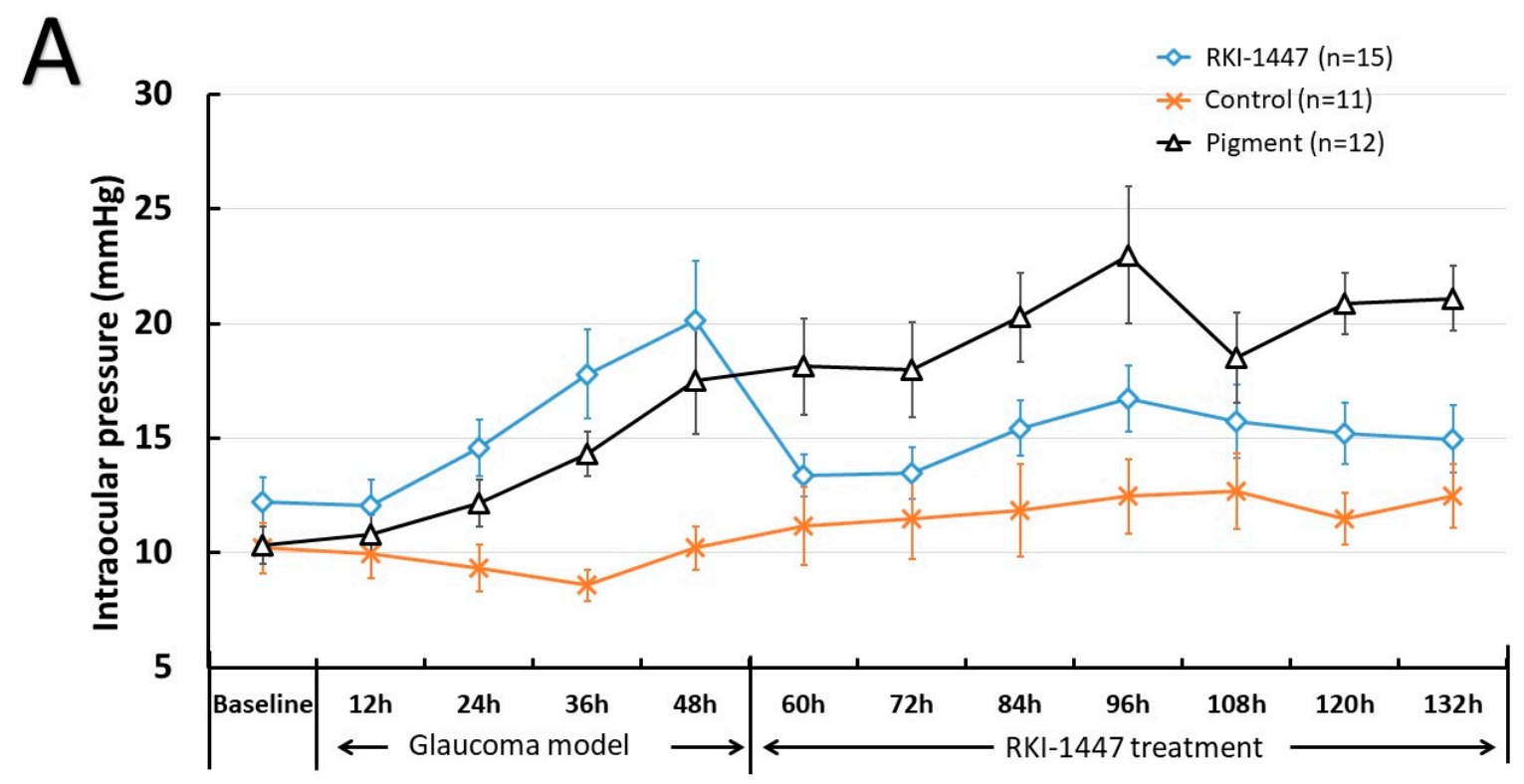

B

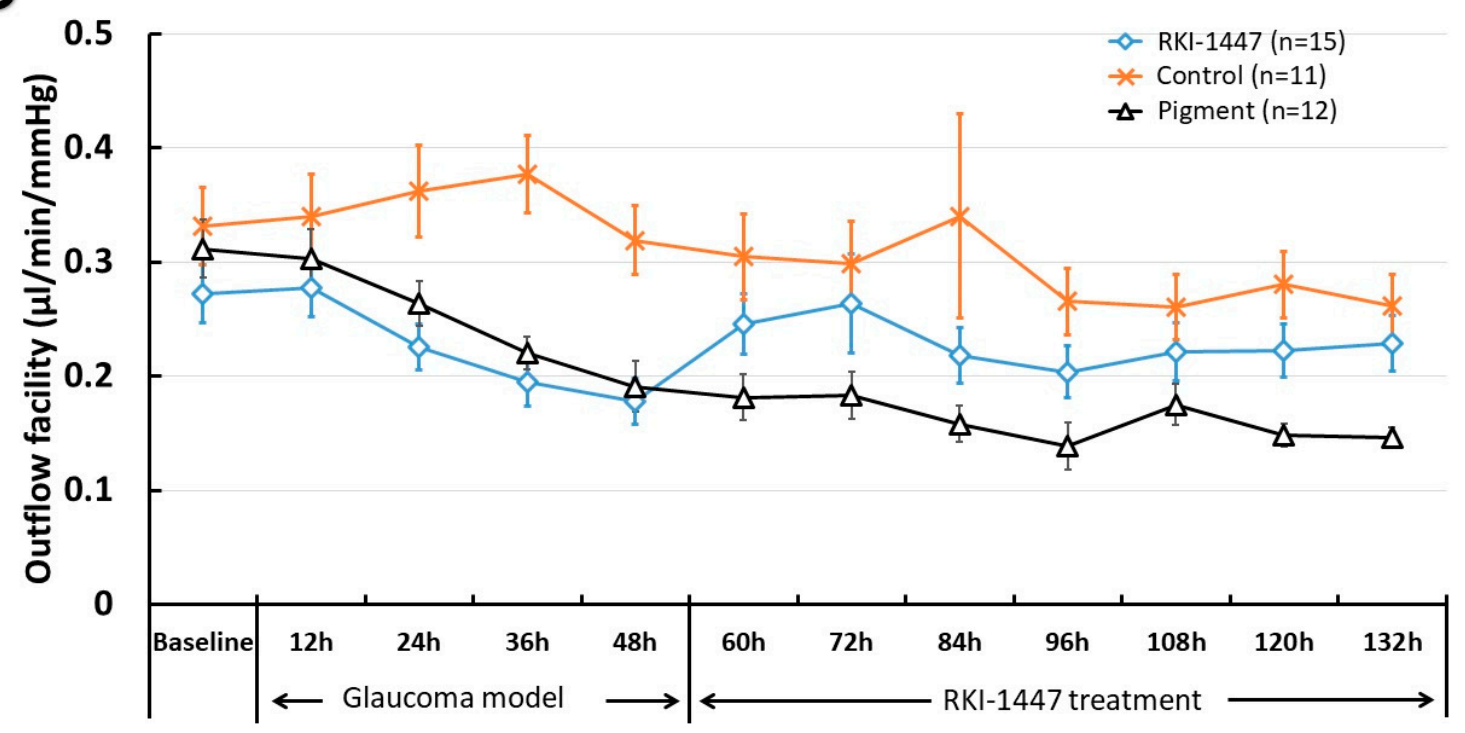

Figure 2. Changes in intraocular pressure and outflow facility by RKI-1447 treatment. (A) IOP at different time points is shown. RKI-1447: blue diamonds, normal control: orange Xs, pigment: black triangles.

Measurements were taken 48 hours after pigment-induced, stable IOP elevation. After treatment with RKI1447 , IOP of the RKI-1447 group decreased significantly from $20.14 \pm 2.59 \mathrm{mmHg}$ to $13.38 \pm 0.91$, while the pigment group remained significantly higher than its baseline (all $\mathrm{P}<0.05$ ). (B) The reduced facility could be increased by RKI-1447. In the pigment group, outflow facility was significantly reduced at all time points in comparison to baseline (all $\mathrm{P}<0.05$ ). 

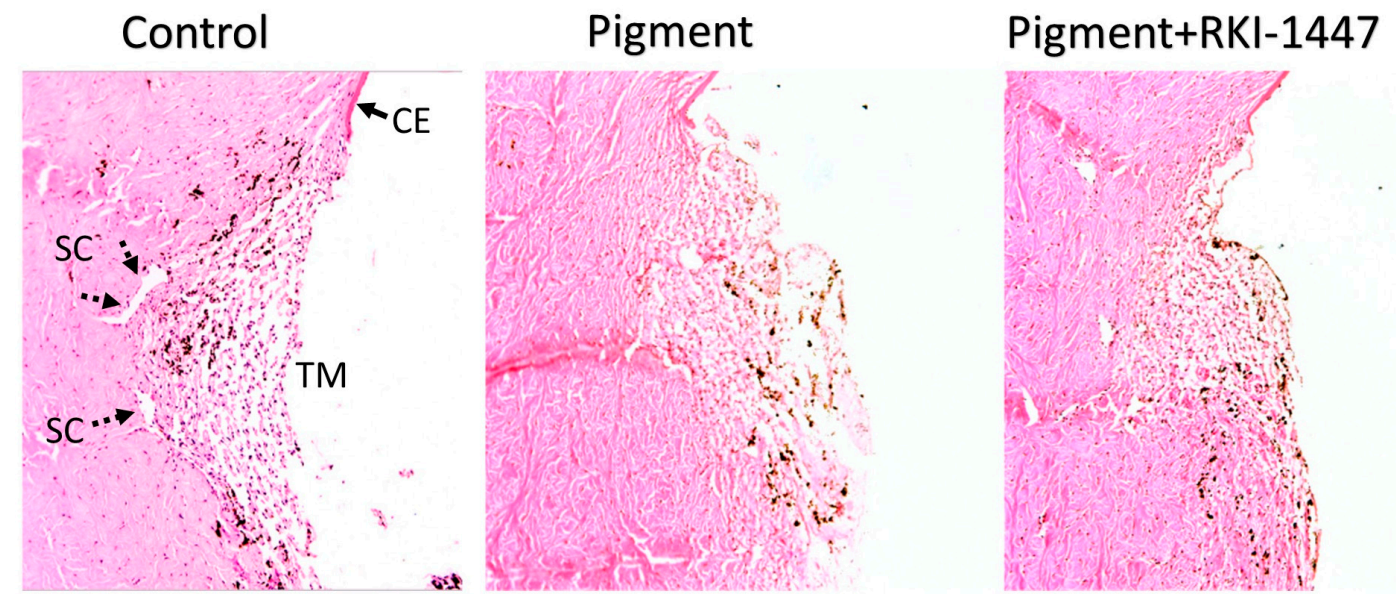

Figure 3. TM histology. The TM in the control group presented as a lightly-pigmented strainer-like tissue, consisting of reticular lamella with TM cells (left). TM of pigment dispersion eyes showed phagocytosed pigment more commonly in the uveal and corneoscleral layers (center: pigment dispersion eye; right: pigment dispersion eye treated with RKI-1447 (right). TM: trabecular meshwork. AP: porcine angular aqueous plexus, with Schlemm's canal-like segments. CE: corneal endothelium. 

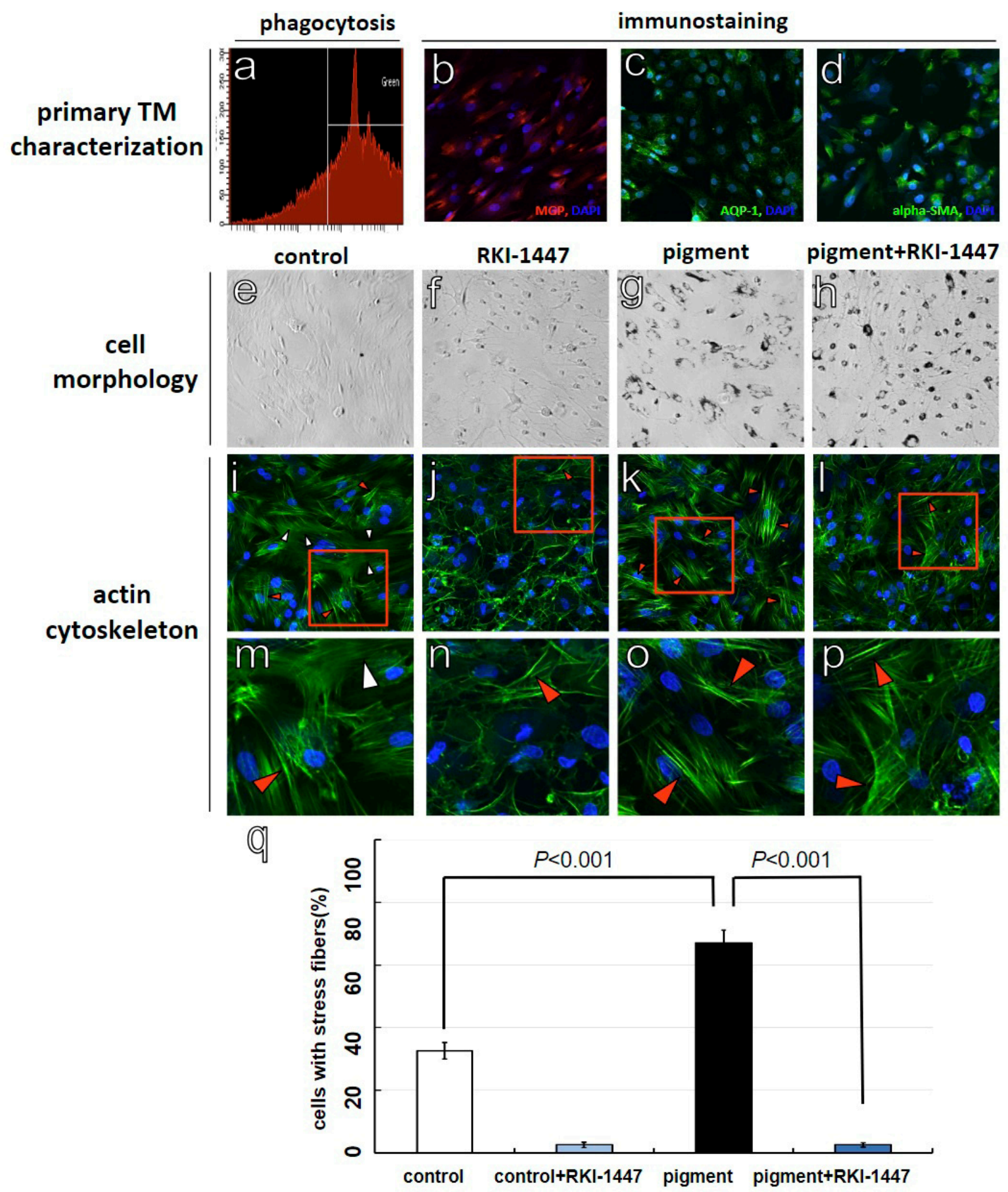

Figure 4. Primary TM characterization, cell morphology, and actin cytoskeleton. Primary porcine TM cells were phagocytically active (4a) and tested positive with the preferential TM-marker antibodies, MGP, AQP-1, and alpha-SMA (4b-d). Pigment exposure did not stimulate a significant morphological change (4e, $f$ ). Cells contracted following RKI-1447 (g, h). RKI-1447 significantly disrupted the actin cytoskeleton regardless of presence or absence of pigment $(P<0.001)(4 \mathrm{i}-\mathrm{I})$. Insets in $4 \mathrm{i}-\mathrm{I}$ correspond to magnified locations in $4 \mathrm{~m}-\mathrm{p}$. Red arrows: F-actin stress fibers, white arrows: non-bundled actin filaments. 

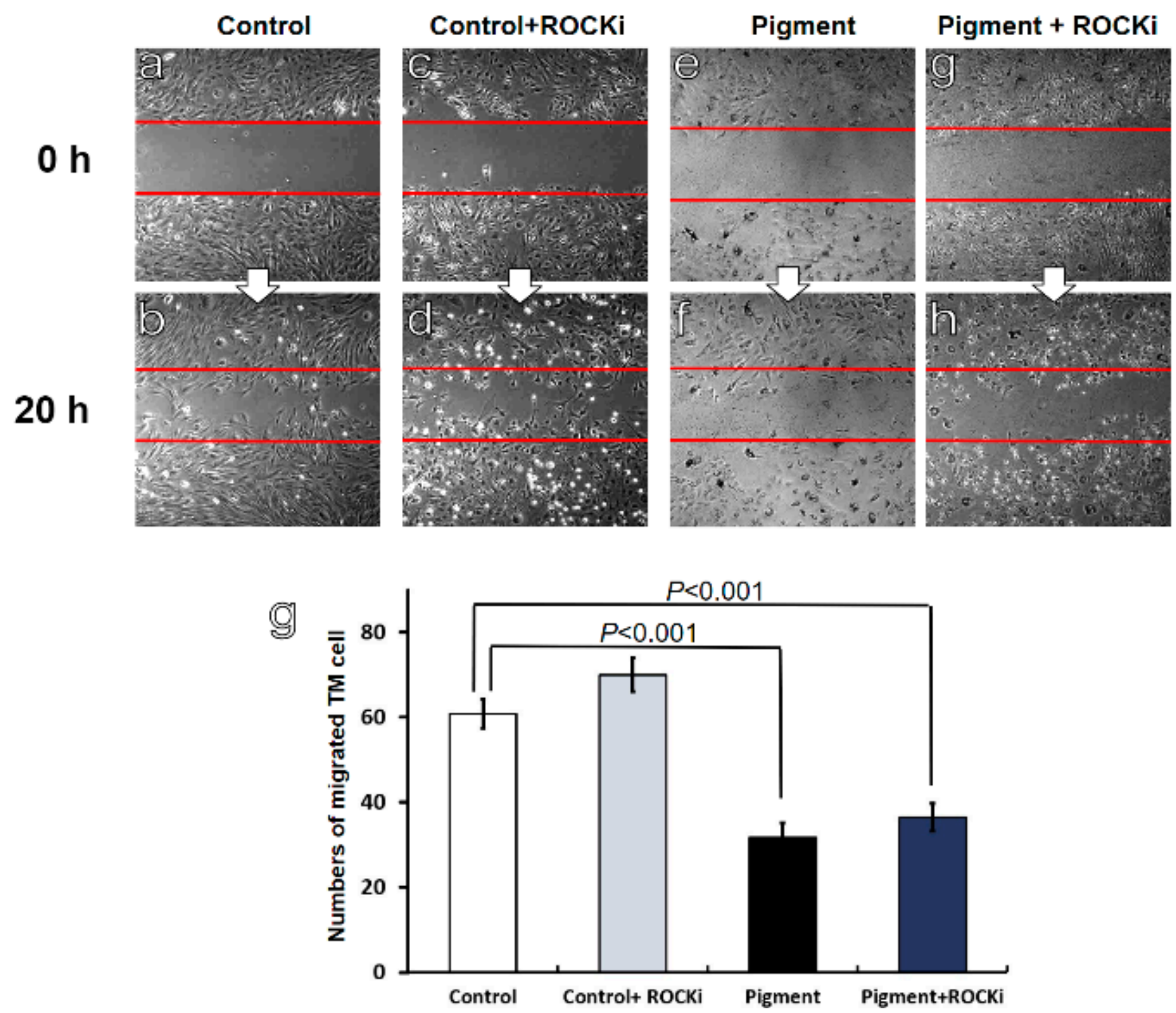

Figure 5. RKI-1447 did not affect TM motility. Motility of TM cells was measured using a scratch assay. Compared to the normal control, pigment exposure resulted in a significant reduction in the number of migrating TM cells $(60.83 \pm 3.46$ in the control versus $31.75 \pm 3.36$ cells in the pigment group, $\mathrm{P}<0.001)$. RKI1447 had no significant effect on normal control TM cells $(P=0.091)$ or pigment dispersion TM cells $(P=0.398)$. 